\title{
Spectral analysis of semi-actively controlled structures subjected to blast loading
}

\author{
C.M. Ewing and C. Guillin \\ Department of Mechanical Engineering, University of Canterbury, Private Bag 4800, \\ Christchurch, New Zealand \\ R.P. Dhakal \\ Department of Civil Engineering, University of Canterbury, Private Bag 4800, \\ Christchurch, New Zealand \\ J.G. Chase \\ Department of Mechanical Engineering, University of Canterbury, Private Bag 4800, \\ Christchurch, New Zealand
}

\begin{abstract}
This paper investigates the possibility of controlling the response of typical portal frame structures to blast loading using a combination of semi-active and passive control devices. A one storey reinforced concrete portal frame is modelled using non-linear finite elements with each column discretised into multiple elements to capture the higher frequency modes of column vibration response that are typical features of blast responses. The model structure is subjected to blast loads of varying duration, magnitude and shape, and the critical aspects of the response are investigated over a range of structural periods in the form of blast load response spectra. It is found that the shape or length of the blast load is not a factor in the response, as long as the period is less than $25 \%$ of the fundamental structural period. Thus, blast load response can be expressed strictly as a function of the momentum applied to the structure by a blast load. The optimal device arrangements are found to be those that reduce the first peak of the structural displacement and also reduce the subsequent free vibration of the structure. Semi-active devices that do not increase base shear demands on the foundations in combination with a passive yielding tendon are found to provide the most effective control, particularly if base shear demand is an important consideration, as with older structures. The overall results are summarised as response spectra for eventual potential use within standard structural design paradigms.
\end{abstract}

Keywords: structural control; semi-active device; blast loading; momentum; free vibration; shear stress; peak displacement; permanent deflection.

† Corresponding author, E-mail: rajesh.dhakal@canterbury.ac.nz 


\section{Introduction}

Blast loads differ from seismic loads in that they excite higher frequency structural modes, rather than only lower or fundamental frequency modes (Yong et al. 2001). Hence, damage to structures from blast loads is also contributed by local response of its components and higher order global response which oscillate with significantly higher frequency than that of the global fundamental mode. Moreover, due to the impulsive nature of the blast load, structural response and the potential damage can be described in two stages. Firstly, the initial impact of the blast produces large shear stresses within the structure, which can cause a structure to collapse. Secondly, after the initial impact of the blast there is a free vibration response, which can produce significant deformations due to the large initial velocity induced by blast. This free vibration phase consists of higher order modes for a short period after the conclusion of the blast load, whereas the later part of the free vibration response is found to consist of gradually decaying response associated with the fundamental global mode (Dhakal and Pan 2003). Thus, structures that survive the initial blast impact can still be severely damaged due to excessive non-linear free vibration displacement in this stage (Dhakal and Pan 2003). Reducing both the initial shear stress and the amount of displacement in the free vibration stage are thus required to reduce the likelihood of failure, and/or permanent damage requiring extensive and costly repair.

The amount of displacement that occurs in the free vibration stage depends on the size of the first displacement peak that results directly from the initial impact of the blast load. If a tendon is braced between two appropriate locations in the structure to control this response, the peak displacement is likely to be reduced. However, this approach also raises the base shear stress in the structure. The tendon may also yield or stretch, reducing its effect on subsequent cycles. Nevertheless, if the structural shear stress remains below levels that cause failure, the tendon will improve the chances of a structure surviving the free vibration stage of the response.

An emerging ideal method of reducing structural displacement during free vibration phase is the use of semi-active devices. Semi-active resetable devices non-linearly alter the stiffness of the structure with stored energy being released as the compressed fluid reverts to its initial pressure, rather than being returned to the structure (Bobrow et al. 2000). These emerging devices have been studied extensively for seismic structural control (Hunt 2002, Barroso et al. 2003, Chase et al. 2004) and have the ability to re-shape hysteretic behaviour to reduce base shear demand in some implementations (Mulligan et al. 2005, Chase et al. 2006, Rodgers et al. 2006a). They also offer the added advantage of being effective on every cycle of the response instead of a limited number. However, they have not been examined for the blast load case.

Research into structural control of blast response of buildings is not extensive. This paper aims to give a broad quantitative assessment of passive and semi-active methods to reduce the likelihood of structural failure due to blast load. The parameters that control the blast load response are first identified. For this purpose, the effect of different blast load pressure wave shapes and intensities are investigated first using a simple structure. Several passive and semi-active methods of reducing the structural response to blast loading for this simple structure are investigated and evaluated in the format of blast load spectra. The primary outcome is to delineate those methods that effectively reduce response while minimising the demand on the foundations and quantify their contribution in a manner that complements existing design methods to better enable potential uptake in the future. 


\section{Analytical background}

\subsection{Structural model}

General structures are best represented by multi-storey, multiple degree of freedom systems. However, to better understand the important features of blast response, a simple single storey portal frame is used in this study. Such a simple model is also an effective and well accepted means of relating the results to design practice and guidelines using a spectral response format.

A non-linear finite element model of a single storey portal frame was created. Due to the nature of blast loads, the modelling technique differed slightly from traditional finite element models of such structures. Blast loads tend to excite the higher frequency modes of the structure on which they act (Yong et al. 2001). It is therefore important that the model is able to capture these higher frequency modes that are excited by the initial short pulse of the blast load, particularly where they represent local excitations of columns or beams. As a result, a traditional finite element model of frames with the column masses lumped at the floors is not appropriate. Instead, the columns mass must be distributed throughout the column. Previous study (Yong et al. 2001) has shown that discretising the column mass into six parts throughout the column is sufficient to accurately model the expected structural response.

The frame used in the analyses in this study is assumed to be made of reinforced concrete. As shown in Fig. 1(a), the frame is 3.6 metres wide and 3.6 metres tall. The beam and column frame members have the same $300 \mathrm{~mm} \times 300 \mathrm{~mm}$ cross-section. The frame is analysed using a non-linear finite element program written in MATLAB. A bilinear hysteretic model, in which the stiffness degrades to $5 \%$ of the initial elastic stiffness after yielding, as shown in Fig. 1(b), is employed for the frame elements used to model the beams and columns. Based on reasonable values of material and geometrical properties of the frame, the yield force and the initial stiffness of these elements are calculated to be $F_{y}=22.5 \mathrm{kN}$ and $K=260 \mathrm{kN} / \mathrm{m}$, respectively. These elements are used so that yielding of the structure could be accurately modelled and captured.

\subsection{Blast load quantification}

During a blast, structures are subjected either to ground shaking (in cases of underground explosion) or to lateral pressure (common for air-blast). In either case, the ground shaking or the

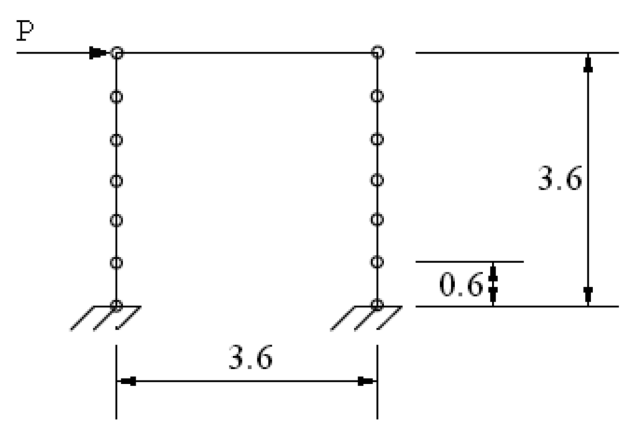

(a) Frame layout

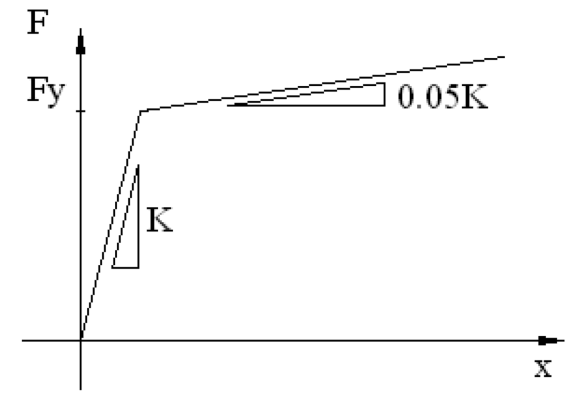

(b) Bilinear hysteretic model

Fig. 1 General model arrangement 

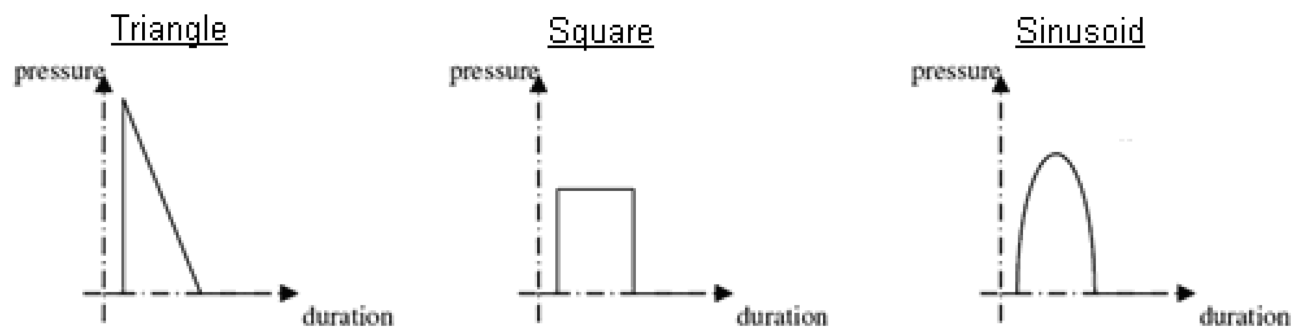

Fig. 2 The 3 blast profiles investigated: triangular, square and sinusoidal

lateral pressure is of an impulsive nature, with a high peak and short duration. In this study, the blast load is modelled as a point load, $P$, acting on the top of the left column of the structure, as shown in Fig. 1(a). The point load, $P$, is in Newtons, which is obtained by multiplying the blastinduced pressure by the area it acts upon, which is assumed to be half the face area.

To understand the dynamics of a blast load acting on the structure, various types of blast load profiles (time history of the pressure/load) are investigated. In this study, a blast load is characterised with three unrelated features: shape of the blast pressure history, the amplitude of the blast pressure, and the duration of the blast pressure. Three different blast load shapes are investigated; triangular, square and sinusoidal, as shown in Fig. 2. Initially, the duration of the blast load is held constant. The amplitude of the blast load is then varied for each blast shape. Subsequent analyses examine the effect of pulse duration.

Fig. 3 shows the variation of the maximum response of the frame (i.e., peak displacement) with respect to different characteristics of the blast loading. To investigate the relationship between structural response and blast input, the blast load shape and duration were held constant and its magnitude or amplitude was varied first. The three different shapes of blast pressure history with a constant duration of 0.034 seconds were applied with varying amplitudes. The results are plotted in Fig. 3(a), which shows that as the amplitude of the blast load increases, the maximum displacement of the frame increases for all three blast shapes. The response of the structure to a square blast load was about twice that of the structure subjected to a triangular blast load, indicating a linear correlation of the peak displacement with the energy input. Next, the amplitude of the blast load was held constant at $350 \mathrm{kPa}$ with the three different shapes, and the duration was then varied up to $t / T=0.5$, the limit for an impulsive load. Holding the amplitude of the blast wave constant and varying the duration produced similar results, as shown in Fig. 3(b). Increasing the duration with constant amplitude also increased the magnitude of the maximum response in proportion to the total energy input.

Overall, the loading profile with the largest area under its amplitude-time curve produced the greatest response. When the three profiles had the same area, they produced exactly the same responses, as shown in Fig. 3(c). This uniqueness holds as long as the duration is much shorter than the structural period; i.e., as long as the applied blast load can be categorised as an impulse. Analysing the units involved, the area under the pressure vs. time plot can be shown to be the momentum input to the system. These results indicate that the response of the structure depends on the amount of momentum applied to it, rather than its shape, rate, amplitude or duration. Hence, the area under the pressure-time plot of the blast load history (i.e., momentum applied) is the only critical parameter that describes the severity of blast loads, and it is the only parameter varied in all subsequent analyses. 


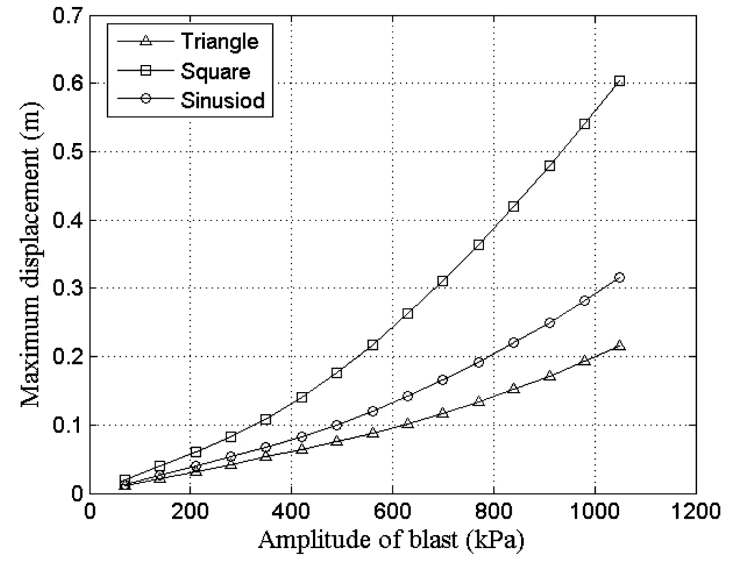

(a) Effect of amplitude (duration $=0.034 \mathrm{~s}$ )

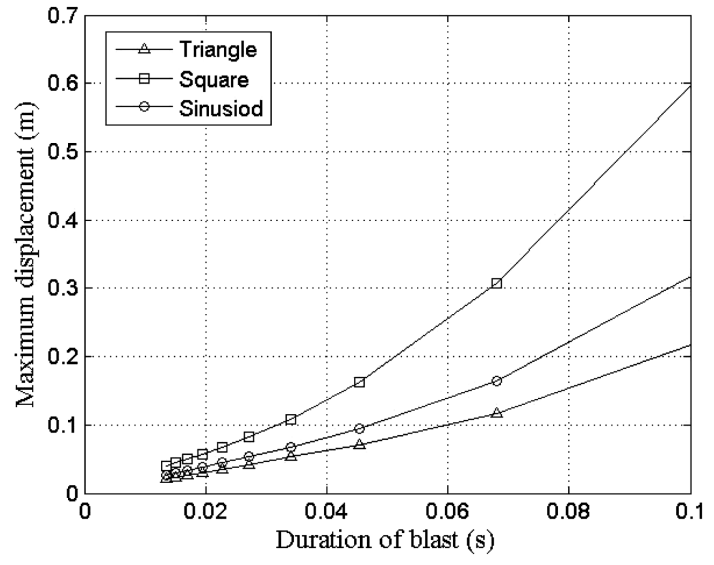

(b) Effect of duration (amplitude $=350 \mathrm{kPa}$ )

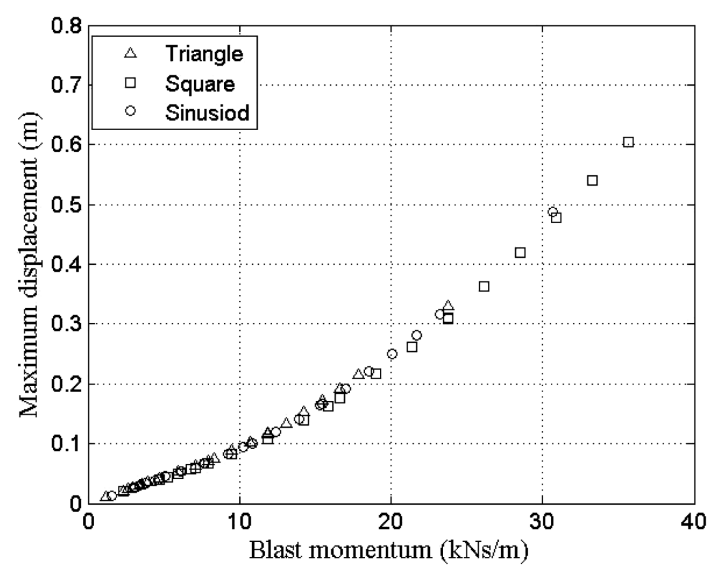

(c) Effect of blast load momentum

Fig. 3 Effect of differing blast loads on a frame

\subsection{Response characteristics}

The typical response of the single storey frame subjected to an impulsive blast load begins with a large initial displacement characterised by significant plastic deformation. This initial deflection depends directly on the momentum of the blast load. Depending on the total momentum applied, the second and third displacement peaks, which are part of the free vibration response, might also include plastic deformation. The free response eventually decays into a linear-elastic response regime. At rest, there is typically a permanent residual deflection that is the net sum of the plastic deformations.

Fig. 4 shows the typical structural response to a blast load. The main points illustrated are the large first peak displacement, which is dependent on the momentum applied to the structure, the second peak on the opposite side, and the residual permanent deflection at the end of the response. The main goal of this research is to develop an effective combination of a passive tendon and/or semi-active control devices to reduce the free vibration and plastic deformation experienced by the structure. 


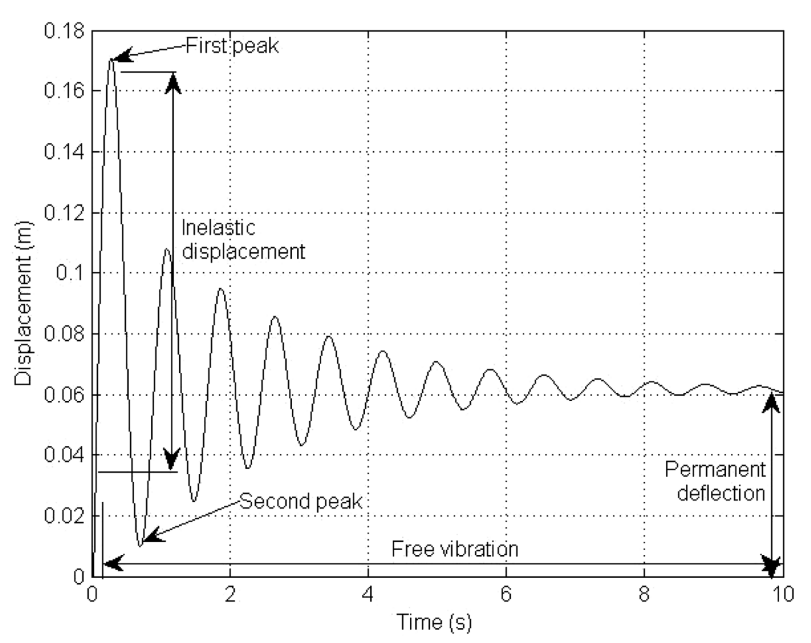

Fig. 4 Typical structural response with the key parameters illustrated

Reducing the initial peak displacement using such control measures also reduces the subsequent free vibration. However, the resistive forces required as the structure moves away from its equilibrium position will also increase the base shear and foundation demands (Chase et al. 2006, Rodgers et al. 2006b). Resisting motion only towards the equilibrium position (from peak amplitudes) can also reduce free vibration and base shear (Rodgers et al. 2006b). Hence, the overall goal is to find the most effective compromise between response reduction and increased base shear demand.

\section{Control devices}

\subsection{Passive device: Tendon}

A tendon is placed diagonally, from the base of one column in the portal frame to the top of the opposite column, to investigate its effectiveness in passively controlling the blast response. The tendon is designed to break once a set yield force is reached. Design specified yielding ensures the base shear in the structure will not reach levels that would cause the structure or its foundation to fail or suffer excessive damage. An optimal tendon design was found, such that the reduction of displacement is maximum and at the same time the base shear does not exceed the capacity of the structure.

\subsection{Semi-active devices}

Semi-active control is an emerging method of limiting structural damage from a range of large environmental loads (Bobrow et al. 2000, Chase et al. 2006, Rodgers et al. 2006b). The main goal of using a semi-active device in this case is to reduce the free vibration after the blast loading has occurred. In this case, the device is anchored to the ground and acts upon the top of the structure. The two types of device considered are a 2-4 device and a 1-4 device. A 1-4 device resists motion between peaks and resets to zero force when the displacement reverses, as shown in Fig. 5(a). On the other hand, a 2-4 device resists only the motion towards the equilibrium (i.e., from the peak 

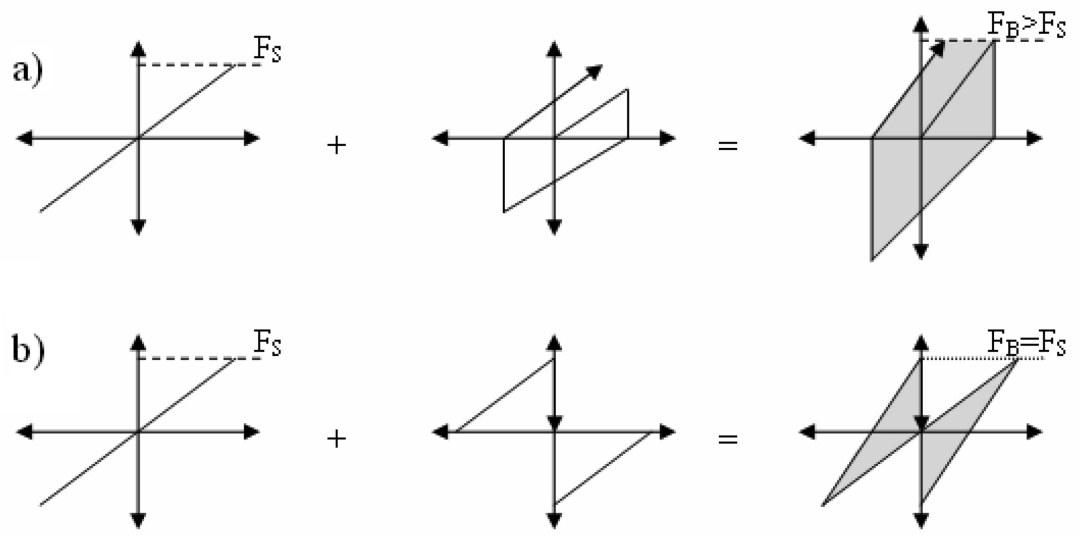

Fig. 5 Semi-active resetable device hysteretic rules for (a) 1-4 device and (b) 2-4 device

displacement in either direction to zero displacement) and thus adds damping only in the $2^{\text {nd }}$ and $4^{\text {th }}$ quadrants, as shown in Fig. 5(b) (Mulligan et al. 2005, Chase et al. 2006). An important difference between these two devices is that a 1-4 device increases the base shear demand whereas a 2-4 device does not. It should also be noted that while 1-4 control resists the first peak response, a 2-4 device will not affect the first peak and only acts subsequently to reduce free vibration.

\subsection{Combinations of the devices and tendon}

Once the effect of each device is understood, they can be appropriately combined to get the best control of the structural response to blast loading. Here, different combinations of the tendon and/or devices are analysed over a range of structural periods to determine the variation in their effectiveness across fundamental structural periods. First, the combination of a tendon and a 2-4 device is investigated. The tendon reduces the effect of the initial blast on the structural response, and the 2-4 device reduces the subsequent free vibration without increasing base shear. Therefore, a combination of the two should be more effective than either individually.

Comparison between a 2-4 device with a tendon and a 2-4 device with a 1-4 device is also made to determine which system performs better. This is to see if a 1-4 device with an appropriate stiffness can effectively replace the tendon. However, the 1-4 resetable device does not return stored energy to the structure unlike a passive tendon does; hence the advantages of the tendon are delineated. Based on the results, guidelines for general design approach for using such device combinations in various structures can be recommended for improving blast load resistance. More specifically, the spectral analyses offer well accepted results that can be more easily integrated into design practice.

\section{Effect of control devices on the structural response}

The following analyses use a specific structure ( $T=1$ second) with specific blast load characteristics (triangular shape, $350 \mathrm{kPa}$ amplitude, 0.034 seconds duration, i.e., $5950 \mathrm{kNs} / \mathrm{m}$ momentum). 


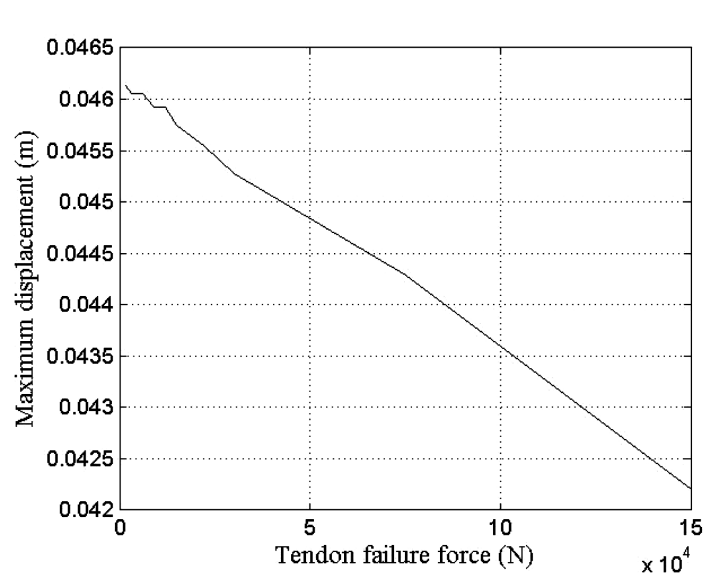

(a)

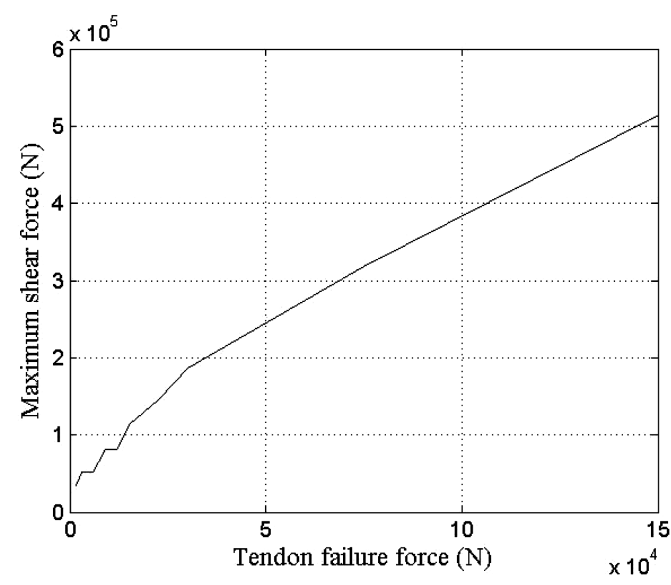

(b)

Fig. 6 Effect of different tendon sizes on: (a) the peak displacement (b) the maximum shear force

\subsection{Passive tendon}

First, analyses are carried out on the frame braced by a tendon with varying sizes. Note that the stiffness of the tendon and the force carried by the tendon before rupture is proportional to its size. Fig. 6 shows the effect of different tendon sizes on two different critical parameters of the structural response; maximum shear force induced and the peak displacement. As can be seen in Fig. 6(a), the maximum displacement is reduced when a tendon is used, and the reduction is more prominent if the tendon size/stiffness increases. As a result of reduction in maximum displacement which happens at the first peak, the subsequent free vibration phase also becomes less prominent and the inelastic deformation as well as the permanent (i.e., residual) deflection is also significantly lower. On the other hand, this reduction in peak displacement comes at a cost of increased base shear, as shown in Fig. 6(b). Overall, the size of the tendon used is found to be critical in controlling the frame response. An increase in the tendon size/stiffness/capacity results in a reduction in the maximum and permanent residual displacements but an increase in the maximum induced shear force.

Therefore, to get the greatest reduction in displacement without failure, the amount of shear force that can be introduced to the structure must be determined, and the tendon needs to be sized accordingly. For this SDOF example structure, a tendon failure force of $100 \mathrm{kN}$ was thought to be optimum as it induces a maximum shear force of $400 \mathrm{kN}$ in the frame which is calculated to be slightly less than the factored shear capacity of the cross section. But, this gives only a nominal reduction of the maximum displacement; from $46 \mathrm{~mm}$ in an uncontrolled case to $43.5 \mathrm{~mm}$ with the tendon. However, if the shear capacity is increased by putting more/closer stirrups or by other strengthening means, one could easily go for a larger tendon which would give further reduction in maximum displacement without threatening the shear capacity.

\subsection{Semi-active devices}

Figs. 7(a) and 7(b) show the effect of a 2-4 device on the blast load response for the same applied momentum, with no tendon. The results show that when using a semi-active $2-4$ device there is a trade off between the permanent deflection and the reduction of the $3^{\text {rd }}$ peak displacement, which is 


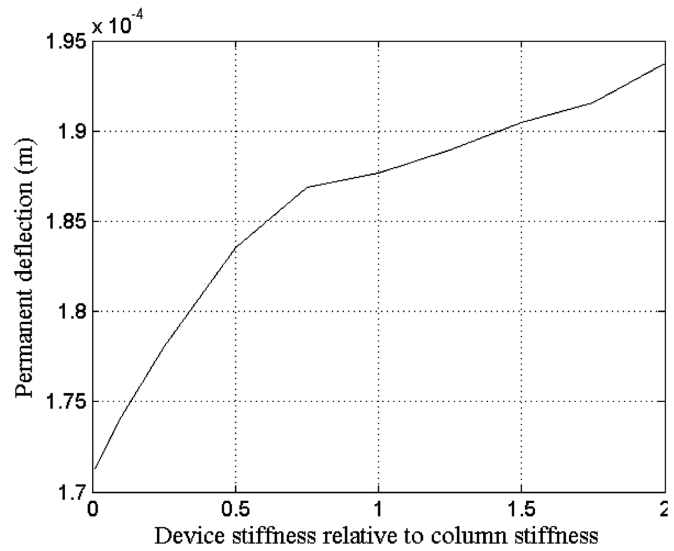

(a)

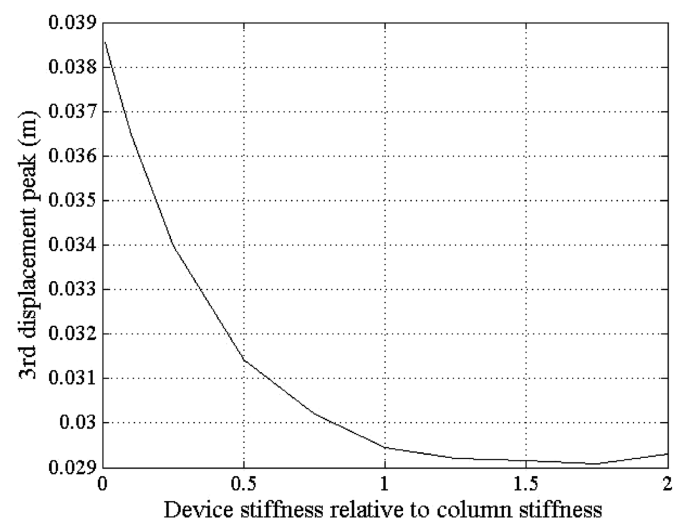

(b)

Fig. 7 Effectiveness of a 2-4 device on the blast response of the frame

thought to be indicative of the free vibration response. However, the permanent deflection is less than $0.2 \mathrm{~mm}$ (see Fig. 7(a)) and any increase in the permanent deflection due to the 2-4 device is negligible regardless of the device stiffness. On the other hand, the reduction in the free vibration response (indicated by the $3^{\text {rd }}$ displacement peak in Fig. 7(b)) is far more significant; close to $25 \%$. This is because a 2-4 device resists only the motion from a peak back towards equilibrium. On the motion after the initial peak, it can thus partially or fully prevent yielding in the opposite direction after the first peak. As can be seen in Fig. 7(b), increasing the device stiffness past that of the column has minimal advantage, as the further reduction in the free vibration response is small.

The difference between the first and third peak displacement can provide a good indication of how long the free vibration phase will last. The closer the $3^{\text {rd }}$ peak to the $1^{\text {st }}$ peak (i.e., the smaller the difference), the longer it will take to decay the response. As a 2-4 device does not affect the $1^{\text {st }}$ peak and reduces the $3^{\text {rd }}$ peak (see Fig. 7(b)), it increases the difference. In other words, a 2-4 device facilitates a rapid decay of the free vibration response phase by resisting motion from the peaks back towards equilibrium.

As explained earlier, the residual permanent deflection increases slightly because the second peak moves closer to the first peak due to reduced yielding back toward the original equilibrium. Increasing the amount of permanent deflection may not be desirable in many cases. Reducing the first displacement peak helps to reduce all permanent deflection. To achieve this without a tendon, a resetable device was set to run as a 1-4 device until the first peak before reverting to a 2-4 device control law for the remaining cycles. A similar scenario was also tested with the 1-4 device acting over 2 displacement peaks. The 1-4 device reduces the first peak of displacement and dissipates energy a tendon does not, but at a cost of also increasing the shear forces acting on the structure.

Fig. 8 shows the reduction in the first displacement peak as the stiffness of the 1-4 device is increased. A significant reduction (more than 20\%) occurs as the device stiffness approaches that of the column stiffness (Rodgers et al. 2006a, 2006b). Above the column stiffness, the rate of reduction slows, as observed previously.

Fig. 9(a) shows the difference between the third peak displacement responses for the three device arrangements, a 2-4 device, a 1-4 device acting over 1 displacement peak and reverting to a 2-4 device, and a 1-4 device acting over two displacement peaks and reverting to a 2-4 device. All the 


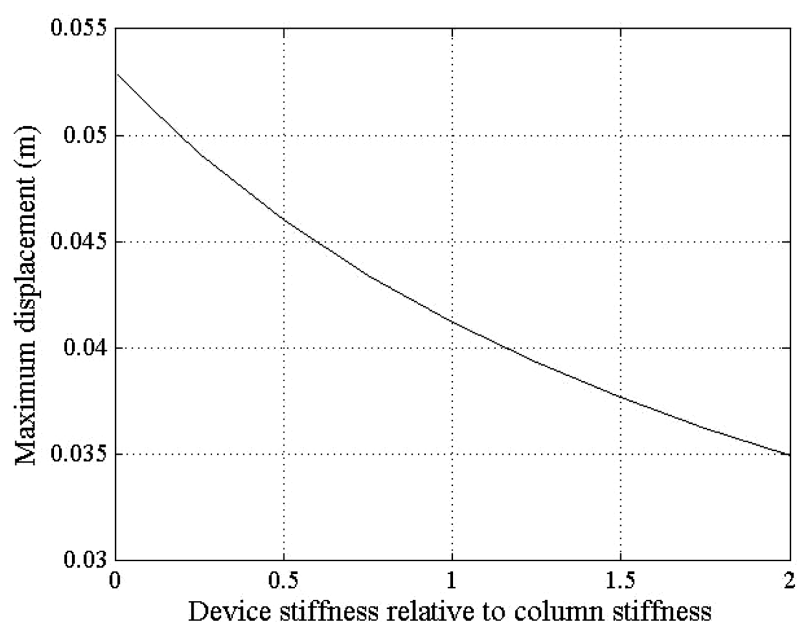

Fig. 8 First peak displacement using a 1-4 device acting until the first displacement peak

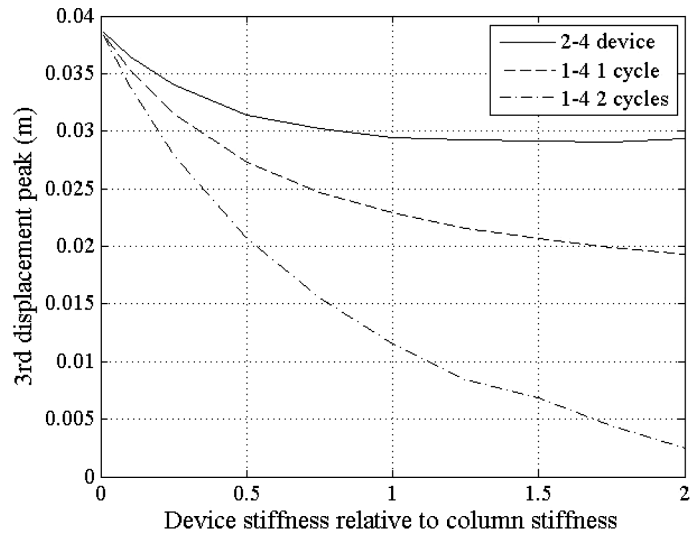

(a) Effect on the third peak

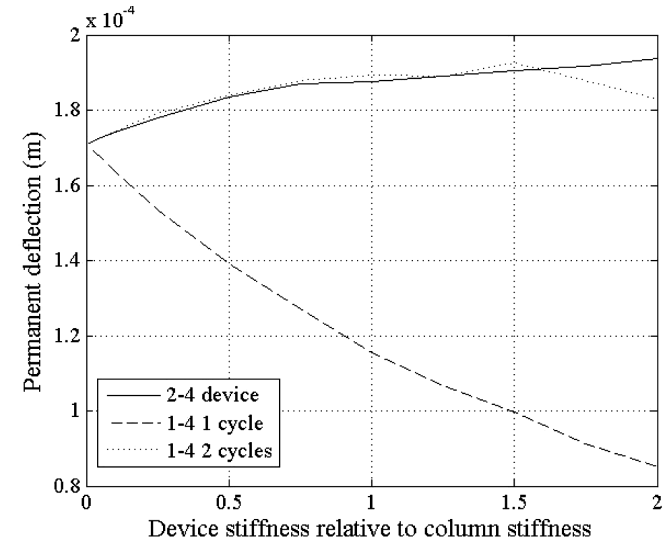

(b) Effect on permanent deflection

Fig. 9 Comparison between the 2-4 device alone and 1-4 device acting until the $1^{\text {st }}$ and $2^{\text {nd }}$ peak with a 2-4 device also acting

semi-active devices are of equal stiffness. As expected, the arrangement with the 1-4 device acting over two peaks performs the best. This result is due to the 1-4 device continuing to act up to and past the point at which the second peak is reached and its greater energy dissipation per cycle (Rodgers et al. 2006a). In the arrangement with the 1-4 device acting over 1 peak, the 1-4 device finishes acting half a cycle before the second peak is reached, making it less effective compared to the arrangement with the 1-4 device acting over two peaks.

Fig. 9(b) shows the variation of permanent displacement with respect to the device stiffness for different combination of 1-4 and 2-4 devices. The 2-4 device acting alone increases the residual deformation, whereas the 1-4 device (until the first or second peak) reverting to a 2-4 device arrangements decrease the permanent displacement to a very different level because of the reduction of the first displacement peak. The arrangement with the 1-4 device acting over two peaks shows larger permanent deflection than when acting until the first peak only because acting over two peaks opposes the structure's tendency to return to its original equilibrium for a longer time period. 


\section{Design spectra}

\subsection{Tendon}

The period of the structure was varied to create a spectral response plot for the structure with a tendon having a yield force of $30 \mathrm{kN}$. The maximum displacement response of the structure with and without the tendon is plotted against the structural period in Fig. 10(a). As the structural period increases, the magnitude of the first displacement peak decreases. As shown in the figure, the reduction of the peak response is as significant as $20 \%$ for $0.5 \mathrm{sec}$ period structures and this reduction becomes more significant for smaller period (stiffer) structures. The third peak displacement provides another indication of how long the subsequent free vibration phase will last. Fig. 10(b) compares the variation of the third peak displacement for structures with different periods with and without a tendon. It is seen that a significant reduction in the third peak displacement (almost $40 \%$ for $0.5 \mathrm{sec}$ structure) can be achieved. Above a period of 2 seconds the reduction in the third peak displacement remains effectively constant. As is obvious from Fig. 10, the effectiveness of the tendon is greatest for structures with lower periods, where higher modes are less excited. Thus, for structural periods greater than 2 or 3 seconds a tendon may not be necessary. A tendon designed for use in blast load mitigation should thus only be considered if the natural period of the structure is less than 2.5 seconds, as this is the period where the tendon starts showing positive effect on the peak displacement and the free vibration response phase.

The permanent deflection response spectra, shown in Fig. 11(a), show a trend similar to the third displacement peak. The tendon effectively stops the structure from yielding in the opposite direction after the first peak has occurred, by reducing the extent of initial yielding and releasing some stored energy when the tendon fails. For periods above 1 second, the permanent displacement is almost non-existent and hence the reduction in the permanent deflection by the tendon is minimal.

None of the previous measurements give an idea of how much total plastic deformation the structure undergoes. Fig. 11(b) shows the total amount of inelastic deformation, or absolute sum of plastic deformation over all cycles, that the structure undergoes in any direction. The tendon reduces the amount of inelastic deformation significantly, particularly for structural periods less than 1 second. As can be seen, the reduction in total plastic deformation (which arguably best represents

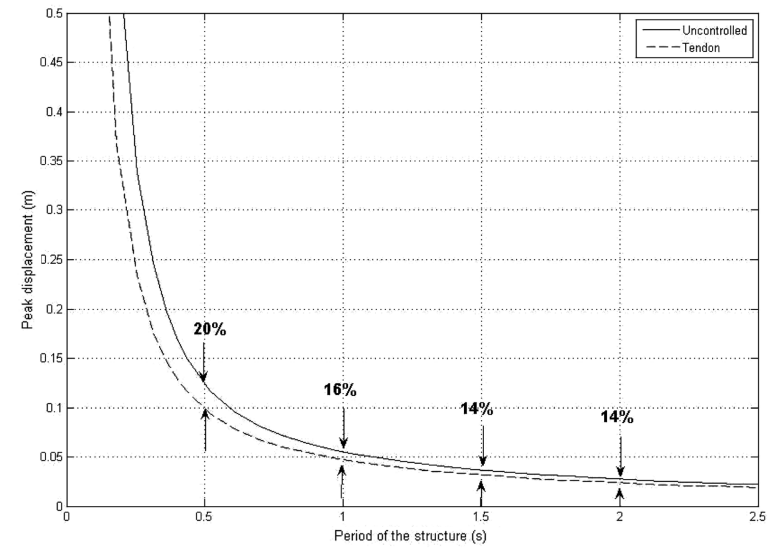

(a) Effect on the first peak

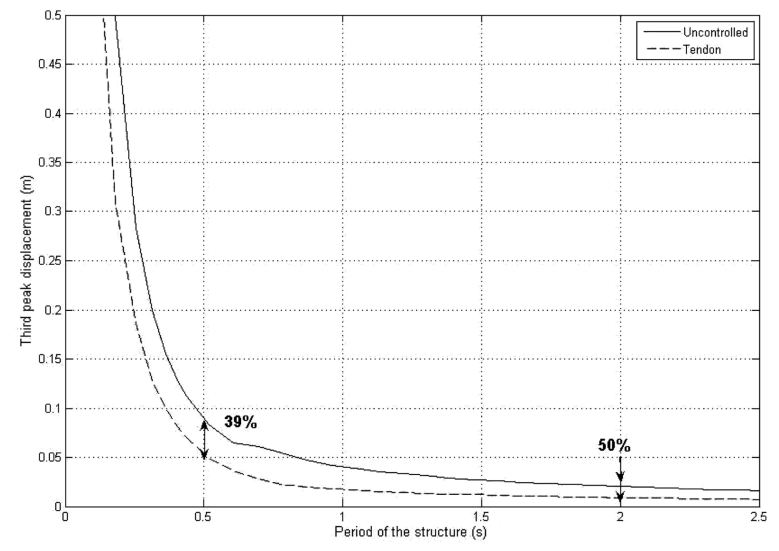

(b) Effect on the third peak

Fig. 10 The effectiveness of using a tendon 


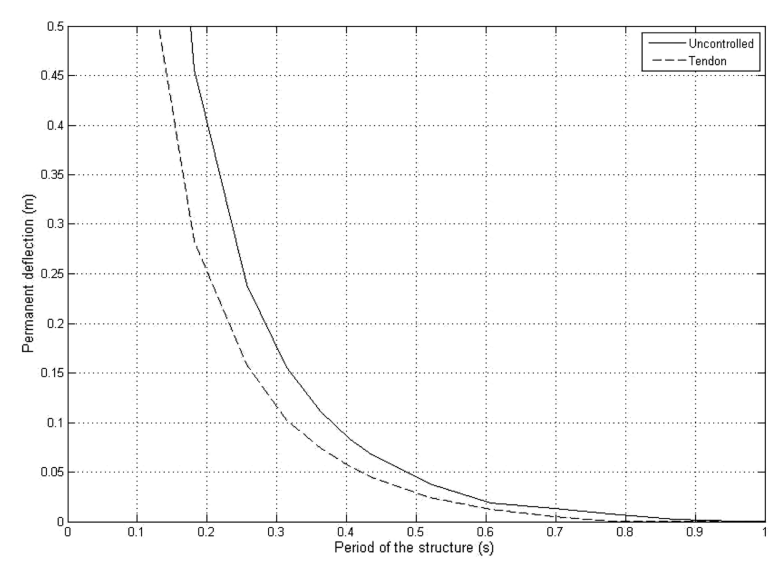

(a) Effect on permanent deflection

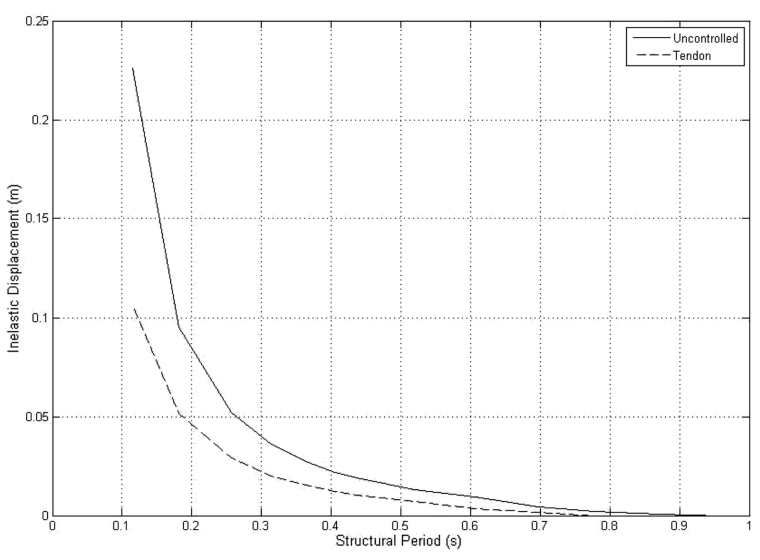

(b) Effect on inelastic displacement

Fig. 11 Effectiveness of a tendon

the damage) is significant with the tendon for stiff structures, whereas more flexible structures (with period more than 1 second), the plastic deformation is non-existent; i.e., the structure does not yield.

\subsection{Semi-active devices}

Three device arrangements were investigated over the same range of structural periods. A 2-4 device alone, a 2-4 device in combination with a 1-4 device that acts only until the first displacement peak, and a 2-4 device in combination with a 1-4 device that acts over the first two displacement peaks are investigated. All devices are assigned the same stiffness equal to the column stiffness.

Fig. 12(a) presents the peak displacement vs structural period results for the uncontrolled structure and the same structure with semi-active control systems. As expected, the 2-4 device acting alone does not reduce the first peak, while by adding a 1-4 device (acting over the $1^{\text {st }}$ or $2^{\text {nd }}$ peak displacements) reduces the first peak the same amount as there is no difference between these two cases until the first peak is reached. Fig. 12(a) shows that the 1-4 device arrangements are more

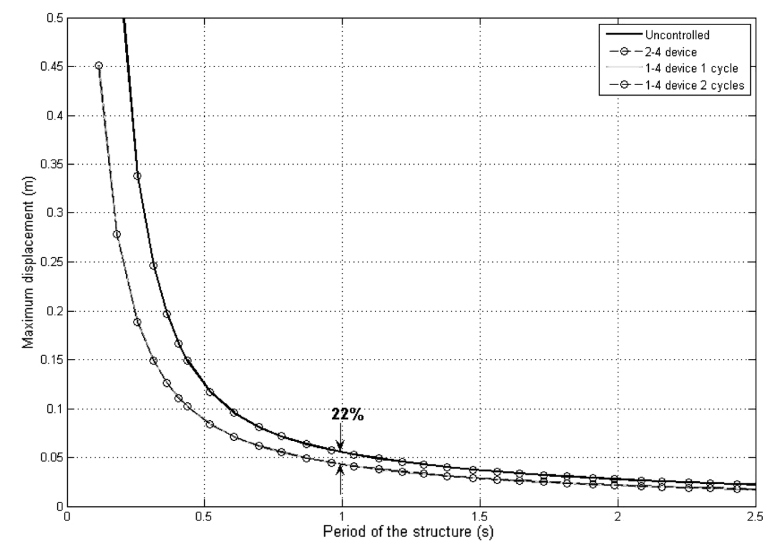

(a) Peak displacement

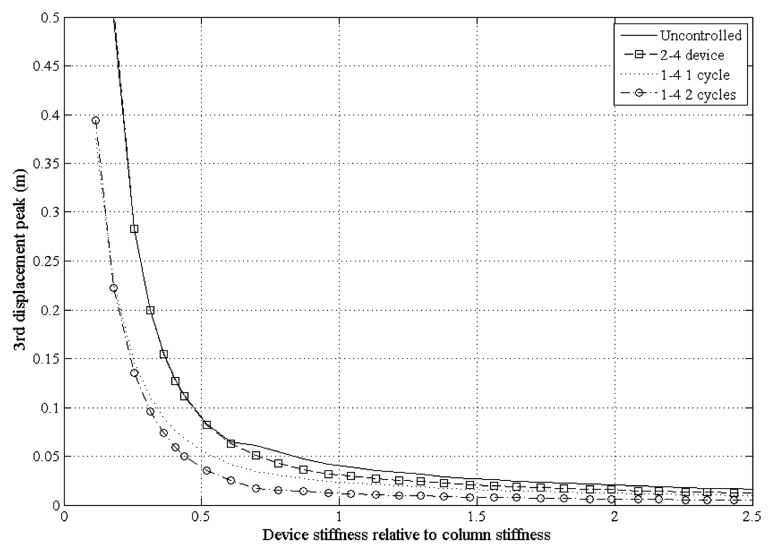

(b) Third peak displacement

Fig. 12 Response spectra with different combinations of semi-active devices 
effective for higher frequency (i.e., smaller period) structures. After a structural period of 2.5 seconds the uncontrolled peak and the controlled peak become almost similar.

Fig. 12(b) shows the third displacement peak over a range of structural periods. The 2-4 device acting alone displays only a minimal difference compared to the uncontrolled case as it has acted only twice by the third peak. The 1-4 device acting over one peak shows a significant decrease in the third displacement peak, which indicates the extent of free vibration response phase. The 1-4 device acting over two peaks shows an even greater decrease. However, both cases appear to have little effect on the response for structural periods greater than 2.5 seconds.

Overall, the 1-4 devices are more effective at lower periods because the first peak for these lower periods dominates the response, whereas for higher periods the first peak is less pronounced. For higher period structures, a 2-4 device alone is desirable due to the response relying less on the first displacement peak. All devices have impact on permanent deflection with the 1-4 devices having mixed effect because it also increases the structure's shear demand. Only 2-4 devices do not increase the base shear demand.

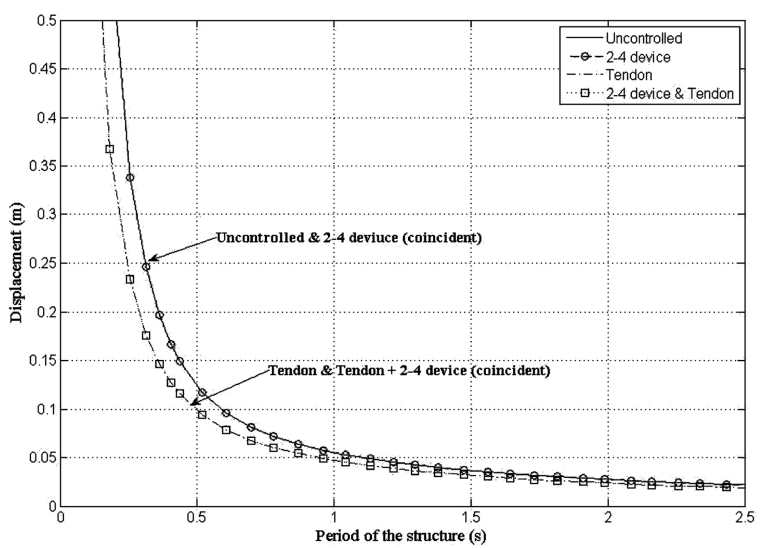

(a) Peak displacement

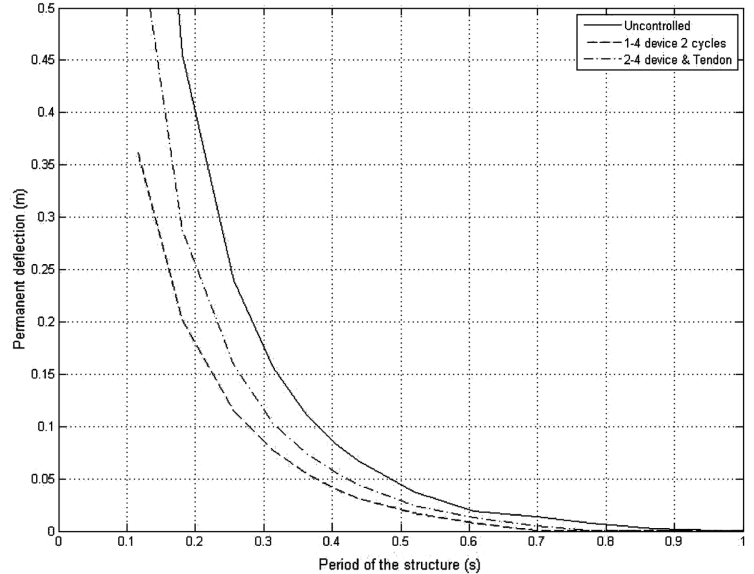

(b) Permanent displacement



(c) Inelastic displacement

Fig. 13 Comparison between the 2-4 device alone, tendon alone and both 


\subsection{Combination of tendon and devices - semi-active and passive solutions}

For structures with periods lower than 2.5 seconds the use of a tendon greatly reduces the overall response of the structure. Semi-active devices can significantly impact the length of the free vibration response. The effectiveness of using a 2-4 device in combination with a tendon is therefore of interest for such structures, particularly where base shear demand is of concern. Fig. 13(a) shows that the effect of this combination on the first peak is no different to that of a tendon alone, which is expected. It is also found that when the tendon is combined with a 2-4 device, the third peak displacement reduces even further than with a tendon alone. This change leads to a slight increase in the permanent deflection, as shown in Fig. 13(b).

Fig. 13(c) compares the amount of inelastic displacement the structure experiences in the uncontrolled, 1-4 to 2-4 device, and the tendon and 2-4 device cases. It is difficult to compare the tendon with the 1-4 device as there is no way to relate the size of each approach/device. However, when comparing the same amount of displacement reduction, the tendon adds less shear demand on the structure as compared with the 1-4 device system. This result indicates that structures with a natural period below 2.5 seconds would benefit most from the use of a tendon, and more so from the use of a tendon and 2-4 device in combination.

\section{Conclusions}

The magnitude, profile or duration of a blast load alone are not individual governing parameters that change structural response to blast loading. Instead, the amount of momentum that the blast load applies to the structure is the parameter that governs the structural response. The response is characterised by an initial large acceleration response to the impact of the blast load with significant plastic deformation, followed by a free vibration phase with smaller oscillations.

The use of a tendon decreased the amount of free vibration by limiting the initial response to the blast loading. The tendon also increased the amount of shear stress in the structure. The amount of shear stress introduced to the structure increases linearly as the size of the tendon is increased. A semi-active 2-4 device reduces the amount of displacement in the free vibration phase of the structure's response to blast loading. As the device only resists motion towards equilibrium, it also effectively increases the amount of permanent deflection. Combining a tendon and a 2-4 device is effective in reducing a structure's total response to blast loads. The tendon reduces the effect of the initial blast impact and the 2-4 device reduces the subsequent free vibration. A 1-4 device in combination with a 2-4 device achieves similar results but with increased base shear. As the period of the structure increases, the effectiveness of both the device and the tendon decreases. As the period of the structure increases above 2.5 seconds, the effect of any system is significantly reduced, and there is little observable difference between the controlled and uncontrolled cases.

\section{References}

Barroso, L.R., Chase, J.G. and Hunt, S.J. (2003), "Resetable smart-dampers for multi-level seismic hazard mitigation of steel moment frames", J. Struct. Con., 10(1), 41-58.

Bobrow, J.E., Jabbari, F. and Thai, K. (2000), “A new approach to shock isolation and vibration suppression 
using a resetable actuator", ASME Transactions on Dynamic Systems, Measurement and Control, 122, 70-573.

Chase, J.G., Barroso, L.R. and Hunt, S.J. (2004), "The impact of total acceleration control for semi-active earthquake hazard mitigation", Eng. Struct., 26(2), 201-209.

Chase, J.G., Mulligan, K.J., Gue, A., Alnot, T., Rodgers, G.W., Mander, J.B., Elliott, R.B., Deam, B.L., Cleeve, L. and Heaton, D. (2006), "Re-shaping hysteretic behaviour using semi-active resetable device dampers", Eng. Struct., 28(10), 1418-1429.

Dhakal, R.P. and Pan, T.C. (2003), "Response characteristics of structures subjected to blasting-induced ground motion", Int. J. Imp. Eng., 28, 813-828.

Hunt, S.J. (2002), Semi-active Smart-dampers and Resetable Actuators for Multi-level Seismic Hazard Mitigation of Steel Moment Resisting Frames, Masters Thesis, Department of Mechanical Engineering, University of Canterbury, Christchurch, New Zealand.

Mulligan, K.J., Chase, J.G., Gue, A., Alnot, T., Rodgers, G.W., Mander, J.B., Elliott, R.E., Deam, B.L., Cleeve, L. and Heaton, D. (2005), "Large scale resetable devices for multi-level seismic hazard mitigation of structures", Proc. $9^{\text {th }}$ International Conference on Structural Safety and Reliability (ICOSSAR 2005), Rome, Italy, June 19-22, 8-pages, ISBN 90-5966-040-4.

Rodgers, G.W., Mander, J.B., Chase, J.G., Mulligan, K.J., Deam, B.L. and Carr, A.J. (2006a), "Re-shaping hysteretic behaviour using resetable devices to customise structural response and forces", 8th US National Conference on Earthquake Engineering (8NCEE), San Francisco, April 18-21, 10-pages.

Rodgers, G.W., Mander, J.B., Chase, J.G., Mulligan, K.J., Deam, B.L. and Carr, A.J. (2006b), "Re-shaping hysteretic behaviour - spectral analysis and design equations for semi-active structures", Earthq. Eng. Struct. Dyn., 36(1), 77-100.

Yong, L., Hao, H., Guowei, M. and Zhou, Y. (2001), "Simulation of structural response under high frequency ground excitation", Earthq. Eng. Struct. Dyn., 30, 307-325. 